\title{
Article \\ Quantum Spacetime, Noncommutative Geometry and Observers
}

\author{
Fedele Lizzi $1,2,3$ (D)
}

1 Dipartimento di Fisica “Ettore Pancini”, Università di Napoli Federico II, 80126 Napoli, Italy; fedele.lizzi@unina.it

2 Istituto Nazionale di Fisica Nucleare (INFN), Sezione di Napoli, 80126 Napoli, Italy

3 Departament de Física Quàntica i Astrofísica and Institut de Cíencies del Cosmos (ICCUB), Universitat de Barcelona, 08028 Barcelona, Spain

Abstract: I discuss some issues related to the noncommutative spaces $\kappa$ and its angular variant $\rho$-Minkowski with particular emphasis on the role of observers.

Keywords: noncommutative geometry; quantum observers; quantum symmetries

\section{Introduction}

Gravity is the theory of (curved) spacetime. Its dynamical variable is spacetime itself, and in fact one way to quantize it is to consider the metric $g^{\mu v}$ as the field to quantize and proceed to build a quantum field theory, on an "ordinary" space. Despite some successes this attempt has not been completely successful, quantum gravity is still an elusive theory. The idea behind the Noncommutative Geometry approach [1-3] to Quantum Gravity is that the object to quantize is spacetime itself, giving rise to a Quantum Spacetime. I will concentrate on kinematics, describing the space by a noncommutative algebra, which can be sometimes described by noncommuting coordinate functions. This is a rather minimal approach which does not do justice to the noncommutative geometry programme, but it will do for purposes of this paper.

Observers play a fundamental role in both general relativity and quantum mechanics. Their role is however subtly different. There is a further ambiguity. Here by observer in general relativity we do not mean a whole sets of clocks and rods (a reference frame), but rather an observer located at a single event, which will be in relations with others. In some sense the observer in this case is synonym with event. Observers are immersed in spacetime, they are its fabric. In quantum mechanics an observer is separated from the phenomenon it wants to describe. Sometime it is classical, i.e., follows different rules from the event. This is not the place to enter the discussion of Schrödinger cats and Wigner friends, it will suffice to notice that the observer is usually considered a classical objet.

The most famous noncommutative space is the one described by noncommuting coordinates, whose commutator is constant. Sometimes it is called DFR (Doplicher, Fredenhagen, Robers) [4] noncommutativity, or Moyal or Gronëwold-Moyal, who introduced the deformed product $[5,6]$ which generalizes this kind of noncommutativity. It also featured in the famous article of Seiberg and Witten [7] on noncommutative geometry and strings. It is described by the commutation relation:

$$
\left[x^{\mu}, x^{\nu}\right]=\mathrm{i} \theta^{\mu \nu} .
$$

Copyright: (C) 2021 by the author Licensee MDPI, Basel, Switzerland. This article is an open access article distributed under the terms and conditions of the Creative Commons Attribution (CC BY) license (https:// creativecommons.org/licenses/by/ $4.0 /)$.

with $\theta$ a constant tensor.

This is a spacetime replica of the quantum phase space canonical commutation relations, with $\hbar$ replaced by $\theta$. This meant that we could use all the experience and technology acquired for quantum mechanics, including the deformed $*$-products above. The unpalatable feature is that this kind of noncommutativity breaks Lorentz invariance, although it maintains translation invariance. This was not a problem for quantum mechanics since 
there is not, in general, a symmetry rotating coordinates into momenta. However, for spacetime Lorentz transformations, spacetime symmetries are a must. This kind breaking of the Lorentz symmetry means not only the presence of a scale, (or scales if the numerical values of the entries of the matrix $\theta$ differ considerably). More cogent is the presence the fact that the constant $\theta$ is an antisymmmetric four by four tensor, which can be expressed as two directions (the analog of electric and magnetic fields for $F_{\mu \nu}$. This implies two fundamental constant directions, a vector and a pseudovector, which would be a fundamental characteristic of our universe. These fundamental directions would show up for example in cosmology, and the recent data pose stringent limits on this. But the problem is not only "phenomenological", it would be bizarre if a quantization of gravity where to prescind from the fundamental symmetries of spacetime.

\section{Quantum Spaces and Their Symmetries}

There is however the possibility that a quantum spacetime will have quantum symmetries, as described by quantum groups, or more precisely Hopf algebras. Quantum Groups and Hopf Algebras developed in parallel to the algebraic/topological approach of Noncommutative Geometry, with several intersections, a classic reviee is [8]. Of particular interest for us is the deformation of the Poincaré Lie algebra which goes under the name of $\kappa$-Poincaré [9-11]. Here I can only mention a few features of it, since I want to concentrate on the role of the observers. It suffices to say that the homogenous space for this quantum symmetry is generated by the commutation rule

$$
\left[x^{0}, x^{i}\right]=\mathrm{i} \lambda x^{i} ;\left[x^{i}, x^{j}\right]=0 .
$$

where $\lambda$ is a dimensionful quantity with the dimension of a length. The relation (2) is often written in terms of $\frac{1}{\kappa}=\lambda$, hence the name. Later I will discuss a space which is a variation on this theme. I will study this space using the usual techniques of quantum mechanics. Let me first briefly recall a well known case study: the Quantum Phase Space of a particle.

Quantum mechanical phase space is a six-dimensional (or in general an even dimensional) space spanned by $\left(q^{i}, p_{i}\right)$. Quantization introduces the commutation relation

$$
\left[q^{i}, p_{j}\right]=\mathrm{i} \hbar \delta_{j}^{i}
$$

The most common representations of position and momenta is operators on $L^{2}\left(\mathbb{R}_{q}^{3}\right)$

$$
\hat{q}^{i} \psi(q)=q^{i} \psi(q) ; \quad \hat{p}_{i} \psi(q)=-\mathrm{i} \hbar \frac{\partial}{\partial q^{i}} \psi(q)
$$

Both the $\hat{q}^{\prime}$ s and $\hat{p}^{\prime}$ s are unbounded self-adjoint operators with a dense domain. The spectrum is the real line (for each $i$ ). We know from the Stone-Von Neumann theorem that, up to unitary transformations, this is the only possible representation. We also know that they have no proper eigenvectors (normalized functions) but improper eigenfunctions: distributions. Since the $\hat{q}^{i \prime}$ s commute it is possible to have a simultaneous improper eigenvector of all of them, these are the Dirac distributions $\delta(q-\bar{q})$ for a particular $\bar{q}$ vector in $\mathbb{R}^{3}$ For a particular momentum $\bar{p}$ the improper eigenfunctions of the $\hat{p}_{i}$ are plane waves $\mathrm{e}^{\mathrm{i} \bar{p}_{i} q^{i}}$. Formally, the eigenvalue equation $\partial_{q} \psi(q)=\alpha \psi(q), \alpha \in \mathbb{C}^{3}$ is solved by $\mathrm{e}^{\alpha \cdot q}$ with a vector $\alpha$.

There is no way that such an exponential can belong to $L^{2}\left(\mathbb{R}^{3}\right)$, therefore also in this case there are no proper eigenfunctions. The operator $\hat{p}$ is unbounded and symmetric, it has a domain of self-adjointness in absolutely continuous functions. This fixes $\alpha$ to have vanishing real part, so that the "function" must be understood in the distributional sense and be well defined on the domain of self-adjointness of the operators. The improper eigenfunctions of momentum are physically interpreted as infinite plane waves of a given frequency. Since plane waves are not vectors of the Hilbert space there is no quantum state for which a measure of the moment would give a precise value. Nevertheless we talk of 
the momentum of a particle in a well defined sense, when a state is a square integrable superposition of plane wave peaked around a given value.

We are representing vectors of the Hilbert space as functions of $q$, this means that we have selected $\hat{q}^{i}$ as a complete set of observables, the description of a quantum state as a function of positions. $|\psi(q)|^{2}$ (normalized) is the density probability to find the particle at position $q$.

The wave function $\psi$ contains also information about the momentum in the phase. Indeed we could have chosen momenta $\hat{p}$ equally well for the complete set of observables. In this basis the wave function $\tilde{\psi}(p)$ is the Fourier transform of the original $\psi$. The fact that the Fourier transform maps square integrable functions into square integrable functions is crucial in this respect. The fact that the Fourier transform is an isometry means that we can use either representation equivalently.

Other choice of complete sets of commuting observables are possible, some with continuous spectrum, like the three number operators along the three directions, or the global number operator, the square of angular momentum and the angular momentum in a given direction.

\section{Observers and Observables for $\kappa$-Minkowski}

Although this is not always clearly stated: observables are related to an observer. Consider now a different observer, for the sake of clarity imagine the second observer rotated or translated with respect to the first one. The second observer will describe the same states with different quantities, it will have its own Hilbert space, set of states and observables. Indeed there are precise rule on how to transform from one observer to the other. The rules are given by the action of a group, represented as unitary operators, on the set of states. Notice also that unavoidably there must be tensor products involved in the process. This is usually not a problem, we know that how to make a unitary transformation from one set to another. But if there are more observers we need, perhaps implicitly, to put together representations. The mathematical object which describes this is the coproduct. Quantum Groups have taught us that there is more than a Lie algebra structure to symmetries. We need extra structure described by Hopf Algebras, which give us the procedure to combine and transform representations. Usually this is done in a cocommutative way, and we do not notice it. The coproduct is primitive, and the full structure appears redundant.

This discussion on quantization of the previous section was a case study, and was performed in a noncommutative phase space, where the parameter/scale of noncommutativity is $\hbar$. The aim of the discussion is however to discuss $\kappa$-Minkowski. This is a deformation of four dimensional spacetime, with different commutation relation and a deformation parameter $\lambda=\frac{1}{\kappa}$. I will consider also this space a quantum space, but I will only consider its kinematical structure, matter will not be involved, nor $\hbar$ will appear. This will allow me to circumvent the known Pauli objection to the existence of a time operator: that such an operator, dual to the Hamiltonian, would have, by Stone-Von Neumann theorem, the same spectrum of the Hamiltonian, i.e. the real line. But his is in contradiction with the requirement that the energy must be limited from below. However the duality time/energy is given by a commutator which only involves $\hbar$, which we are neglecting.

We are considering (special) relativistic spaces, the speed of light $c$ is necessary to have time $x^{0}$ to have the same dimensions as the spatial coordinates, and it must of course be finite. It is impossible to separate this space from it symmetry: The Poincaré group in primis, which we have to represent as operators on some concrete Hilbert space. I will first look for a representation of the $x^{\mu}$ on square integrable function of position, $L^{2}\left(\mathbb{R}^{3}\right)$. In other words I choose the $x^{i}$ as a complete set of observables. In this case the $x^{i}$ are multiplicative operators:

$$
\hat{x}^{i} \psi(x)=x^{i} \psi(x) .
$$


Time instead, not commuting with the $x^{i}$, is represented by a dilation operator:

$$
\hat{x}^{0} \psi(x)=\mathrm{i} \lambda\left(\sum_{i} x^{i} \partial_{x^{i}}+\frac{3}{2}\right) \psi(x)=\mathrm{i} \lambda\left(r \partial_{r}+\frac{3}{2}\right) \psi(x) .
$$

where $r=\sqrt{x^{i} x_{i}}$. The $3 / 2$ factor is necessary to make the operator symmetric. It has a domain of self-adjointness in absolutely continuous functions. This is not the unique representation of the $x^{\mu}$ on $L^{2}\left(\mathbb{R}^{3}\right)$ which satisfies the commutation relations. These are catalogued in [12]. The most general realisation for $x^{0}$ symmetric is:

$$
\begin{aligned}
\hat{x}^{i} & =x^{i} A\left(\mathrm{i} \lambda \partial_{0}\right) \\
\hat{x}^{0} & =x^{0} B\left(\mathrm{i} \lambda \partial_{0}\right)+\mathrm{i} \lambda C\left(\mathrm{i} \lambda \partial_{0}\right)\left(r \partial_{r}+\frac{3}{2}\right) .
\end{aligned}
$$

with the three functions $A, B$ and $C$ which satisfy the constraints $\frac{A^{\prime}}{A} B=(1-C)$ and $C(0)=A^{\prime}(0)+1$ finite, and the boundary conditions $A(0)=1, B(0)=0$. Our choice is to have all quantities constant: $A=1, B=0$ and $C=1$.

The $\kappa$-Minkowski space is spatially isotropic, this suggests that a polar basis is more appropriate. We can rewrite therefore the relations (2) as:

$$
\left[\hat{x}^{0}, \cos \theta\right]=\left[\hat{x}^{0}, \mathrm{e}^{\mathrm{i} \varphi}\right]=0,\left[x^{0}, r\right]=\mathrm{i} \lambda r .
$$

And the uncertainty relation becomes

$$
\Delta x^{0} \Delta r \geq \frac{\lambda}{2}|\langle r\rangle|
$$

We know that the spectrum of $r$ is the positive half-line, with improper eigenfunctions the $\delta(r)$, i.e., localisation of the state at a given distance from the origin. Instead $\theta$ and $\varphi$ are not good operators, but we know we can expand the angular part in spherical harmonics, which is tantamount to consider in the complete set the square of the angular momentum and one of its components. The fact that the angular part is not affected by the commutation relations suggests that issues relating with spin and statistics may not affect observers in this case. This will not be true for the other kinds of noncommutativity considered below.

Let me now find the spectrum of the time operator. First notice that monomials in $r$ are formal solutions of the eigenvalue problem:

$$
\mathrm{i} \lambda\left(r \partial_{r}+\frac{3}{2}\right) r^{\alpha}=\mathrm{i} \lambda\left(\alpha+\frac{3}{2}\right) r^{\alpha}=\lambda_{\alpha} r^{\alpha}
$$

The eigenvalues are real if and only if $\alpha=-\frac{3}{2}+\tau$.

with $-\infty<\tau<\infty$ a real number. Where previously we have seen that for momentum we had plane waves, in this case we have the following distributions:

$$
T_{\tau}=\frac{r^{-\frac{3}{2}-\mathrm{i} \tau}}{\lambda^{-\mathrm{i} \tau}}=r^{-\frac{3}{2}} \mathrm{e}^{-\mathrm{i} \tau \log \left(\frac{r}{\lambda}\right)} .
$$

The distribution has the correct dimension of a length ${ }^{3 / 2}$, the factor of $\lambda$ is there to avoid taking the logarithm of a dimensional quantity. Since $\lambda$ is a natural scale for the model, its choice is natural, but not unique. Whereas for quantum phase space we had as complete set of observables either three $q$ or three $p$, connected by a Fourier transform, for $\kappa$ Minkowski we have either $(r, \theta, \varphi)$ or $(\tau, \theta, \varphi)$, and they are connected by polar version of a Mellin transform:

$$
\psi(r, \theta, \varphi)=\frac{1}{\sqrt{2 \pi}} \int_{-\infty}^{\infty} \mathrm{d} \tau r^{-\frac{3}{2}} \mathrm{e}^{-\mathrm{i} \tau \log \left(\frac{r}{\lambda}\right)} \widetilde{\psi}(\tau, \theta, \varphi)
$$




$$
\widetilde{\psi}(\tau, \theta, \varphi)=\frac{1}{\sqrt{2 \pi}} \int_{0}^{\infty} \mathrm{d} r r^{\frac{1}{2}} \mathrm{e}^{\mathrm{i} \tau \log \left(\frac{r}{\lambda}\right)} \psi(r, \theta, \varphi) .
$$

The functions $|\psi|^{2}$ and $|\widetilde{\psi}|^{2}$ are dual to each other, in the sense that the former is interpreted as the probability density to find the particle in a particular radial and angular position given by $r, \theta, \varphi$. Dually, the modulus square of $\widetilde{\psi}$ is the probability to find the particle at angular position $\theta, \varphi$ at the time $\tau$. The fact that time and the radial coordinate coordinate do not commute means that it is possible to precisely localize a state in space, at the price of not knowing anything about its time localisation. And conversely, a sharp localisation in time implies complete delocalization as far the distance from the origin is concerned. In [13-15] there are various examples of localised/delocalised states.

It is useful to have an idea of the dimensional quantities involved. If we call $t$ the eigenvalue of the time operator $\frac{x^{0}}{c}$, then $\tau=t \frac{c}{\lambda}$. The scale is set by $\frac{c}{\lambda}$, a dimensional quantity with the dimensions of the inverse of a time, a frequency. If we choose for $\lambda$ the Planck length then $\frac{c}{\lambda} \sim 2 \cdot 10^{43} \mathrm{~Hz}$. In other words if $t=1 \mathrm{~s}$, then $\tau=2 \cdot 10^{43}$, an extremely large number. If instead $t$ is of the order of Planck time, then $\tau \sim 1$.

So far we have been treating the origin as a special point. Does this mean that somewhere in the universe there is "the origin". A special position in space singled out by the $\kappa$-God? Implicitly in our discussion, when we were referring to states, we were assuming the existence of an observer measuring the localisation of states. This observer singles out a special point, the one in which he is located: the origin. This observer can measure with absolute precision its position. Subjective, operational "here" and "now" make sense. Is it however impossible for this observer to localise with absolute precision distant states, if some information about the time of the event is to be retained. This is a consequence of the particular noncommutativity of $\kappa$-Minkowski. What about other observers? A different observer will be in general Poincaré transformed, i.e. translated, rotated and boosted. These operations are usually performed with an element of the Poincaré group. But now we have $\kappa$-Poincaré! We should require invariance under the transformation $x^{\mu} \rightarrow x^{\prime \mu}=\Lambda^{\mu}{ }_{v} \otimes x^{v}+a^{\mu} \otimes 1$. of [16])

The coordinate functions on the group are noncommutative, they are (in the basis

$$
\begin{gathered}
{\left[a^{\mu}, a^{v}\right]=\mathrm{i} \lambda\left(\delta^{\mu}{ }_{0} a^{v}-\delta^{v}{ }_{0} a^{\mu}\right), \quad\left[\Lambda^{\mu}{ }_{v}, \Lambda^{\rho}{ }_{\sigma}\right]=0} \\
{\left[\Lambda^{\mu}{ }_{v}, a^{\rho}\right]=\mathrm{i} \lambda\left[\left(\Lambda^{\mu}{ }_{\sigma} \delta^{\sigma}{ }_{0}-\delta^{\mu}{ }_{0}\right) \Lambda^{\rho}{ }_{v}+\left(\Lambda^{\sigma}{ }_{\nu} \delta^{0}{ }_{\sigma}-\delta^{0}{ }_{v}\right) \eta^{\mu \rho}\right] .}
\end{gathered}
$$

Notice in particular that translations are now noncommuting. With the same commutation relations of the coordinates. Their coproduct, antipode and counit are

$$
\begin{aligned}
\Delta\left(a^{\mu}\right) & =a^{v} \otimes \Lambda^{\mu}{ }_{v}+1 \otimes a^{\mu} \\
\Delta\left(\Lambda^{\mu}{ }_{v}\right) & =\Lambda^{\mu}{ }_{\rho} \otimes \Lambda^{\rho}{ }_{v} \\
S\left(a^{\mu}\right) & =-a^{v}\left(\Lambda^{-1}\right)^{\mu}{ }_{v} \\
S\left(\Lambda^{\mu}{ }_{v}\right) & =\left(\Lambda^{-1}\right)^{\mu}{ }_{v} \\
\varepsilon\left(a^{\mu}\right) & =0 \\
\varepsilon\left(\Lambda^{\mu}{ }_{v}\right) & =\delta^{\mu}{ }_{v} .
\end{aligned}
$$

We represented the $\kappa$-Minkowski algebra as operators. But in doing so we had implicitly chosen an observer. In order to take into account the fact that there are different observers we enlarge the algebra (and consequently the space) to include the locations, i.e. the coordinates, of the observers. We call this new set of states as $\mathcal{P}_{\kappa}$. The Hilbert space as well has to take into account the state of the observers, which are identified not only by their relative position, but also by their orientation, or rather the orientation of their reference frame. Hence the Hilbert space will now comprise not only functions on spacetime (either functions of $r$ or $\tau$ ), but also functions of the $a^{\prime}$ s and $\Lambda^{\prime}$ s. 
We can represent the $\kappa$-Poincaré group faithfully as

$$
\begin{aligned}
a^{\rho}= & -\mathrm{i} \frac{\lambda}{2}\left[\left(\Lambda^{\mu}{ }_{\sigma} \delta^{\sigma}{ }_{0}-\delta^{\mu}{ }_{0}\right) \Lambda^{\rho}{ }_{\nu}+\left(\Lambda^{\sigma}{ }_{\nu} \delta^{0}{ }_{\sigma}-\delta^{0}{ }_{\nu}\right) \eta^{\mu \rho}\right] \Lambda^{v}{ }_{\alpha} \frac{\partial}{\partial \omega^{\mu}{ }_{\alpha}} \\
& +\mathrm{i} \frac{\lambda}{2}\left(\delta^{\rho}{ }_{0} q^{i} \frac{\partial}{\partial q^{i}}+\delta^{\mu}{ }_{i} q^{i}\right)+\frac{1}{2} \text { h.c. }
\end{aligned}
$$

where $\omega$ are the parameters of the Lorentz transformation, and the $\Lambda$ 's are represented as multiplicative operators. We have therefore that, like spacetime, the space of observers is also noncommutative, and that the noncommutativity is only present in the translation sector.

We now explore this space of observers, seen seeing them as states. First consider a transformed observer, also located at the origin. The transformation is connecting the two observers is the identity transformation, and we connect it to the counit of the Hopf algebra. Define $|o\rangle_{\mathcal{P}}$ with the property:

$$
\mathcal{P}\langle o|f(a, \Lambda)| o\rangle_{\mathcal{P}}=\varepsilon(f),
$$

with $f(a, \Lambda)$ a generic noncommutative function of translations and Lorentz transformation matrices, and $\varepsilon$ the counit. This state describes the Poincaré transformation between two coincident observers. The state is such that all combined uncertainties vanish. Coincident observers are therefore a well-defined concept in $\kappa$-Minkowski spacetime.

A change of observer will transform $x^{\mu} \rightarrow x^{\prime \mu}=\Lambda^{\mu}{ }_{v} \otimes x^{v}+a^{\mu} \otimes \mathbb{1}$ and primed and unprimed coordinates correspond to different observers. Identifying $x$ with $\mathbb{1} \otimes x$ we generate an extended algebra $\mathcal{P} \otimes \mathcal{M}$ which extends $\kappa$-Minkowski by the $\kappa$-Poincaré group algebra. This algebra takes into account position states and observables. Remember that, just as we cannot sharply localize position states, neither we can sharply localize where the observer is. Since Lorentz transformations commute among themselves, we can however say if two observers are just rotated with respect to each other. Different observers will identify directions in a unique way without problems.

We can build the action of the position, translation and Lorentz transformations operator on generic functions of all those variables. To simplify notations let us consider $1+1$ dimensions. In this case there are only two position coordinates, two translations coordinates and one Lorentz transformation parametrized by the rapidity $\xi$.

The relations among the various quantities are:

$$
\Lambda_{0}^{0}=\Lambda_{1}^{1}=\cosh \xi, \Lambda_{1}^{0}=\Lambda_{0}^{1}=\sinh \xi,
$$

and

$$
\left[a^{0}, a^{1}\right]=\mathrm{i} \lambda a^{1}, \quad\left[\xi, a^{0}\right]=-\mathrm{i} \lambda \sinh \xi, \quad\left[\xi, a^{1}\right]=\mathrm{i} \lambda(1-\cosh \xi) .
$$

The action on $\mathcal{P}$ is

$$
a^{0}=\mathrm{i} \lambda q \frac{\partial}{\partial q}+\mathrm{i} \lambda \sinh \xi \frac{\partial}{\partial \xi}, a^{1}=q+\mathrm{i} \lambda(\cosh \xi-1) \frac{\partial}{\partial \xi},
$$

States (non entangled) will be objects of the kind $|g\rangle \otimes|f\rangle$. In particular $|g\rangle \otimes|o\rangle$ is a pure translation of the state at the origin.

The new observer measures coordinates with $x^{\prime}$. The expectation values on (normalised) transformed state is

$$
\left\langle x^{\prime \mu}\right\rangle=\left\langle g\left|\otimes\left\langle o\left|x^{\prime \mu}\right| g\right\rangle \otimes\right| o\right\rangle=\left\langle g\left|\Lambda^{\mu}{ }_{\nu}\right| g\right\rangle\left\langle o\left|x^{\nu}\right| o\right\rangle+\left\langle g\left|a^{\mu}\right| g\right\rangle\langle o \mid o\rangle,
$$

We get:

$$
\left\langle x^{\prime \mu}\right\rangle=\left\langle g\left|a^{\mu}\right| g\right\rangle,
$$

The expectation value of the transformed coordinates is completely defined by translations. This is natural, the different observers are comparing positions, not directions. 
In general

$$
\left\langle x^{\prime \mu_{1}} \ldots x^{\prime \mu_{n}}\right\rangle=\left\langle g\left|a^{\mu_{1}} \ldots a^{\mu_{n}}\right| g\right\rangle\langle o \mid o\rangle=\left\langle g\left|a^{\mu_{1}} \ldots a^{\mu_{n}}\right| g\right\rangle .
$$

Poincare transforming the origin state $|o\rangle$ by a state with wavefunction $|g\rangle$ in the representation of the $\kappa$-Poincaré algebra, the resulting state will assign, to all polynomials in the transformed coordinates the same expectation value as what assigned by $|g\rangle$ to the corresponding polynomials in $a^{\mu}$. In other words, the state $x^{\prime \mu}$ is identical to the state of $a^{\mu}$.

All uncertainty in the transformed coordinates $\Delta x^{\prime \mu}$ is introduced by the uncertainty in the state of the translation operator, $\Delta a^{\mu}$. It is also possible to see that the uncertainty of states increases with translation. I can summarise saying that all observers can sharply localise states in their vicinity, and cannot localise states far away from them. The apparent paradox of a state badly localisable by Alice, but which is well localised by Bob, is that Bob herself is badly localised by Alice, and of course viceversa.

All this is qualitatively perfectly compatible with the principle of relative locality [17], which however starts in a quite different context: curved momentum space. In this analysis instead momentum does not appear explicitly, although it is present in the symmetry. The space of momenta in this context has been studied in [15]. Note however that the construction may run into troubles for multiparticles states, unless the noncommutativity is light-like [18], a case not discusse here.

One of the tenets of Quantum Mechanics is that the observer is classical, usually macroscopic, and that therefore we "know" how to deal with them. In quantum gravity this may not be the case. While it is true that the smallness of the Planckian constants suggests this, there may be amplifying effects, and conceptual aspects to deals with. The group algebra approach, where the parameters of the Poincaré transformations do not commute is the key to understand the observer-dependent transformations relating different frames belonging to a noncommutative algebra. Hence the localisability limitations. Alternatively, the deformation can be seen as a deformation of the tensor product. This is evident in the case of a Drinfeld twist, and I give another example, based on such a twist.

\section{4. $\rho$-Minkowski and Discrete Time}

We now pass to the discussion of the following noncommutative space, which we call $\rho$-Minkowski, defined by the commutation relations:

$$
\left[x^{0}, x^{1}\right]=-\mathrm{i} \lambda x^{2} ;\left[x^{0}, x^{2}\right]=\mathrm{i} \lambda x^{1} ;\left[x^{0}, x^{3}\right]=0 ;\left[x^{1}, x^{j}\right]=0 .
$$

This form of noncommutativity has a long history, [19-26], more recentlyit was studied in [27-29] who called $\rho$ what I call here $\lambda$, hence the name. I will present here mainly the results in [30] and particularly in [31].

A similar version of noncommutative spaces can be can be built in which $x^{0}$ and $x^{3}$ are exchanged. I will not discuss this variant here. Details can be found in [30].

Just like we did for $\kappa$-Minkowski and polar coordinates, it is useful to express the commutation relations (24) in cylindrical coordinates $(t, \rho, z, \varphi)$

$$
"[t, \varphi]=\mathrm{i} \lambda " ;[t, z]=[t, \rho]="[\rho, \varphi] "=[\rho, z]=0 .
$$

Note that I have put some of the commutators in inverted commas. These commutation relations are to be understood better. We can repeat the previous analysis, but take into account that the angular variables are not good observables. This explains the inverted commas. A better expression would be $[r, Y(\varphi)]=0$, where $Y$ is an operator generated by well defined functions of $\varphi$.

In this case the uncertainty will be between time and the angular variable. And one should definitely resist the temptation to write:

$$
\Delta t \Delta x \leq \frac{\pi}{2}
$$


In the $\{\rho, z, \varphi\}$ basis $t$ is represented by the derivation operator $-\mathrm{i} \lambda \partial_{\varphi}$.

The crucial issue is that this operator, which basically the third component of the angular momentum $L_{z}$, has discrete spectrum!

Just as in the previous case we wish to express wav functions and states in two alternative basis. As before one basis is given by functions of position. Then we have the other basis, that when we consider time as one of operators of the complete set. The basis in which time is diagonals is given this time by the Fourier series. Representing the fact that the operator has discrete spectrum. The eigenstates of momentum are $\mathrm{e}^{\mathrm{i} n \varphi}$, and they are completely delocalised in $\varphi$. On the other hand, a state completely localised in $\varphi$, given by a $\delta$, which requires a superposition with equal weights of all eigenvalues of time.

$$
\delta(\varphi)=\frac{1}{2 \pi} \sum_{n=-\infty}^{\infty} \mathrm{e}^{\mathrm{i} n \varphi} .
$$

After a time measurement, which has given as result $n_{0} \lambda$, the system is in the eigenstate $\mathrm{e}^{\mathrm{i} n_{0} \varphi}$. A slightly uncertain state uses a great number of Fourier modes to built a state peaked around some time, then the corresponding uncertainty is the angular variable is given by the fact that only a finite set of elements of the basis are available. For $\lambda$ Planckian of the quantum of time (also called a chronon), is $5.39 \cdot 10^{-44} \mathrm{~s}$. The most accurate measurement of time is $\sim 10^{-19} \mathrm{~s}$. Heuristically the superposition of $10^{35}$ quanta of time is needed. If we approximate $\delta$ by the Dirichlet nucleus

$$
\delta_{N}=\sum_{n=-N}^{N} \mathrm{e}^{\mathrm{i} n \varphi}=\frac{1}{2 \pi} \frac{\sin \left(N+\frac{1}{2}\right) \varphi}{\sin \frac{N}{2} \varphi} .
$$

Then $N \sim 10^{35}$. In this case the first zero of the nucleus is at $\varphi \sim 10^{-35}$. We may assume this to be the uncertainty in an angle determination. To translate this as an uncertainty in position we need $\rho$. For the radius of the observable universe $\left(10^{26} \mathrm{~m}\right)$ the uncertainty is of the order of one metre. Certainly not a large uncertainty for such a distant state!

Again we should worry about symmetries. Is this all pervading clicking a feature of our universe? Is time translation definitely lost? Putting time on a lattice may be disturbing. Self-adjointness come to the rescue. Anybody who has studied the Aharonov-Bohm experiment knows that the momentum operator on a compact domain is a rich operator. It is self-adjoint on periodic functions, but is also self-adjoint on functions periodic up to a phase. In this case the eigenfunctions are $\mathrm{e}^{\mathrm{i}(n+\alpha) \varphi}$. The differences between states is unchanged, and the effect is a rigid shift. This however means that a different choices of self-adjointess domains. Time translations are undeformed, and two time translated observers will be in different, but equivalent domains. In order to compare their results the two observers, again, have to compare representations, and this is ruled by a coproduct. This time the rotation group is deformed. This may have consequences for the connection between spin and statistics, which may not be related as in the usual way. This issue deserves further scrutiny.

The important ingredient which allow us to study efficiently the Hopf algebra is that this kind of noncommutativity can be built with a Drinfeld twist. The twist is a map from the tensor product of a Lie algebra times itself. The algebra is intended represented over functions. In particular the twist $\mathcal{F}$ must be invertible, and satisfy a cocycle condition:

$$
(\mathcal{F} \otimes 1)(\Delta \otimes i d) \mathcal{F}=(1 \otimes \mathcal{F})(i d \otimes \Delta) \mathcal{F},
$$

with $\Delta(X)=X \otimes 1+1 \otimes X$ the primitive coproduct for the element $X$ in the Lie algebra. What the twist does is to modify the tensor product, so that the pointwise product, which is map from the tensor functions of functions into functions becomes a deforms one. Given 
$\mu: f \otimes g \rightarrow f \cdot g$ the standard pointwise multiplication in $C^{\infty}(M)$, the deformed product of functions is then defined according to

$$
f \star g:=\mu \circ \mathcal{F}^{-1}(f \otimes g) .
$$

The twist is further supplemented with the normalization condition

$$
\mu(\epsilon \otimes 1) \mathcal{F}=\mu(1 \otimes \epsilon) \mathcal{F}=1 .
$$

The Drinfeld twist relevant for $\varrho$-Minkowski is:

$$
\begin{gathered}
\mathcal{F}(x, y)=\exp \left\{-\frac{i \lambda}{2}\left(\partial_{y^{0}}\left(x^{2} \partial_{x^{1}}-x^{1} \partial_{x^{2}}\right)-\partial_{x^{0}}\left(y^{2} \partial_{y^{1}}-y^{1} \partial_{y^{2}}\right)\right)\right\} \\
=\exp \left\{\frac{i \lambda}{2}\left(\partial_{y^{0}} \partial_{\varphi_{x}}-\partial_{x^{0}} \partial_{\varphi_{y}}\right)\right\} .
\end{gathered}
$$

This deforms the Hopf algebra as

$$
\begin{aligned}
\Delta P_{3}= & P_{3} \otimes 1+1 \otimes P_{3}, \\
\Delta P_{0}= & P_{0} \otimes 1+1 \otimes P_{0}, \\
\Delta P_{1}= & P_{1} \otimes \cos \left(\frac{\lambda}{2} P_{0}\right)+\cos \left(\frac{\lambda}{2} P_{0}\right) \otimes P_{1}+P_{2} \otimes \sin \left(\frac{\lambda}{2} P_{0}\right)-\sin \left(\frac{\lambda}{2} P_{0}\right) \otimes P_{2}, \\
\Delta P_{2}= & P_{2} \otimes \cos \left(\frac{\lambda}{2} P_{0}\right)+\cos \left(\frac{\lambda}{2} P_{0}\right) \otimes P_{2}-P_{1} \otimes \sin \left(\frac{\lambda}{2} P_{0}\right)+\sin \left(\frac{\lambda}{2} P_{0}\right) \otimes P_{1}, \\
\Delta M_{01}= & M_{01} \otimes \cos \left(\frac{\lambda}{2} P_{0}\right)+\cos \left(\frac{\lambda}{2} P_{0}\right) \otimes M_{01}+M_{02} \otimes \sin \left(\frac{\lambda}{2} P_{0}\right)-\sin \left(\frac{\lambda}{2} P_{0}\right) \otimes M_{02} \\
& -P_{1} \otimes \frac{\lambda}{2} M_{12} \cos \left(\frac{\lambda}{2} P_{0}\right)+\frac{\lambda}{2} M_{12} \cos \left(\frac{\lambda}{2} P_{0}\right) \otimes P_{1} \\
& -P_{2} \otimes \frac{\lambda}{2} M_{12} \sin \left(\frac{\lambda}{2} P_{0}\right)-\frac{\lambda}{2} M_{12} \sin \left(\frac{\lambda}{2} P_{0}\right) \otimes P_{2}, \\
\Delta M_{02}= & M_{02} \otimes \cos \left(\frac{\lambda}{2} P_{0}\right)+\cos \left(\frac{\lambda}{2} P_{0}\right) \otimes M_{02}-M_{01} \otimes \sin \left(\frac{\lambda}{2} P_{0}\right)+\sin \left(\frac{\lambda}{2} P_{0}\right) \otimes M_{01} \\
& -P_{2} \otimes \frac{\lambda}{2} M_{12} \cos \left(\frac{\lambda}{2} P_{0}\right)+\frac{\lambda}{2} M_{12} \cos \left(\frac{\lambda}{2} P_{0}\right) \otimes P_{2} \\
& +P_{1} \otimes \frac{\lambda}{2} M_{12} \sin \left(\frac{\lambda}{2} P_{0}\right)+\frac{\lambda}{2} M_{12} \sin \left(\frac{\lambda}{2} P_{0}\right) \otimes P_{1}, \\
\Delta M_{03}= & M_{03} \otimes 1+1 \otimes M_{03}-\frac{\lambda}{2} P_{3} \otimes M_{12}+\frac{\lambda}{2} M_{12} \otimes P_{3}, \\
\Delta M_{12}= & M_{12} \otimes 1+1 \otimes M_{12}, \\
\Delta M_{13}= & M_{13} \otimes \cos \left(\frac{\lambda}{2} P_{0}\right)+\cos \left(\frac{\lambda}{2} P_{0}\right) \otimes M_{13}+M_{23} \otimes \sin \left(\frac{\lambda}{2} P_{0}\right)-\sin \left(\frac{\lambda}{2} P_{0}\right) \otimes M_{23} \\
\Delta M_{23}= & M_{23} \otimes \cos \left(\frac{\lambda}{2} P_{0}\right)+\cos \left(\frac{\lambda}{2} P_{0}\right) \otimes M_{23}-M_{13} \otimes \sin \left(\frac{\lambda}{2} P_{0}\right)+\sin \left(\frac{\lambda}{2} P_{0}\right) \otimes M_{13} .
\end{aligned}
$$

With this twist we can build a covariant $\star$ product, and proceed to the study of field, and gauge, theories, as well as the determination of all relations defining the Hopf algebra:

$$
(f \star g)(x)=\left.\mathcal{F}^{-1}(y, z) f(y) g(z)\right|_{x=y=z}=f g-\frac{\mathrm{i} \lambda}{2}\left(\partial_{\varphi} f \partial_{0} g-\partial_{0} f \partial_{\varphi} g\right)+O\left(\lambda^{2}\right) .
$$

which deforms the addition of momenta

$$
e^{-\mathrm{i} p \cdot x} \star e^{-\mathrm{i} q \cdot x}=e^{-\mathrm{i}(p+\star q) \cdot x},
$$

where

$$
p+\star q=R\left(q_{0}\right) p+R\left(-p_{0}\right) q
$$


and

$$
R(t) \equiv\left(\begin{array}{cccc}
1 & 0 & 0 & 0 \\
0 & \cos \left(\frac{\lambda t}{2}\right) & \sin \left(\frac{\lambda t}{2}\right) & 0 \\
0 & -\sin \left(\frac{\lambda t}{2}\right) & \cos \left(\frac{\lambda t}{2}\right) & 0 \\
0 & 0 & 0 & 1
\end{array}\right) .
$$

With this is it possible to build a field theory. In particular we looked at $\phi^{4}$ Euclidean scalar theory. The usual arena to look for phenomena like ultraviolet/infrared mixing.The deformed conservation of momenta gives a deformation of the vertex but not of the propagator. This is because the $\delta$ of conservation of momentum involves the $\star$ sum of momenta given by the $R$ matrix (37). One consequence is that decays are not anymore back to back, details can be found in [30].

To conclude: the main message I wished to convey is that quantum gravity will require Quantum Spacetime. But also that Quantum Spacetime in turn requires quantum observers. This is of course true for quantum phase space as well. There we became (more or less) used to deal with the contradictions of the quantum/classical interaction. We learned how to deal with noncommuting observables for example. But a quantum spacetime will pose further challenges and other layers to our understanding, in this respect see [32].

Funding: This research received no external funding.

Institutional Review Board Statement: Not applicable.

Informed Consent Statement: Not applicable.

Data Availability Statement: Not applicable.

Acknowledgments: I would like first of all to thank the organizers of the conference The Quantum and The Gravity, and my collaborator in the work I presented: M. Dimitrijevic Ciric, N. Konjik, M. Kurkov, M. Manfredonia, F. Mercati, T. Poulain and P. Vitale. I acknowledge support from the INFN Iniziativa Specifica GeoSymQFT and the State Agency for Research of the Spanish Ministry of Science and Innovation through the "Unit of Excellence Maria de Maeztu 2020-2023" award to the Institute of Cosmos Sciences (CEX2019-000918-M) and from PID2019-105614GB-C21 and 2017-SGR-929 grants.

Conflicts of Interest: The author declares no conflict of interest.

\section{References}

1. Connes, A. Noncommutative Geometry; Academic Press: Cambridge, MA, USA, 1995.

2. Landi, G. An Introduction to Noncommutative Spaces and Their Geometry; Springer: Berlin/Heidelberg, Germany, 1997 ; Volume 51. [CrossRef]

3. Gracia-Bondia, J.M.; Varilly, J.; Figueroa, H. Elements of Noncommutative Geometry; Birkhauser: Boston, MA, USA, 2001.

4. Doplicher, S.; Fredenhagen, K.; Roberts, J.E. The Quantum structure of space-time at the Planck scale and quantum fields. Commun. Math. Phys. 1995, 172, 187. [CrossRef]

5. Moyal, J.E. Quantum mechanics as a statistical theory. Proc. Camb. Phil. Soc. 1949, 45, 99. [CrossRef]

6. Groenewold, H.J. On the Principles of elementary quantum mechanics. Physica 1946, 12, 405. [CrossRef]

7. Seiberg, N.; Witten, E. String theory and noncommutative geometry. J. High Energy Phys. 1999, 9, 32. [CrossRef]

8. Majid, S. Foundations of Quantum Group Theory; Cambridge University Press: Cambridge, UK, 1995. [CrossRef]

9. Lukierski, J.; Nowicki, A.; Ruegg, H. Real forms of complex quantum anti-De Sitter algebra U-q(Sp(4:C)) and their contraction schemes. Phys. Lett. 1991, 271, 321. [CrossRef]

10. Lukierski, J.; Nowicki, A.; Ruegg, H. New quantum Poincaré algebra and k deformed field theory. Phys. Lett. 1992, $293,344$. [CrossRef]

11. Majid, S.; Ruegg, H. Bicrossproduct structure of kappa Poincaré group and noncommutative geometry. Phys. Lett. 1994, 334, 348-354. [CrossRef]

12. Meljanac, S.; Stojic, M. New realizations of Lie algebra kappa-deformed Euclidean space. Eur. Phys. J. C 2006, 47, 531. [CrossRef]

13. Lizzi, F.; Manfredonia, M.; Mercati, F.; Poulain, T. Localization and Reference Frames in $\kappa$-Minkowski Spacetime. Phys. Rev. 2019, 99, 085003. [CrossRef]

14. Lizzi, F.; Manfredonia, M.; Mercati, F. Localizability in $\kappa$-Minkowski spacetime. Int. J. Geom. Meth. Mod. Phys. 2020, 17, 2040010. [CrossRef] 
15. Lizzi, F.; Manfredonia, M.; Mercati, F. The momentum spaces of $\kappa$-Minkowski noncommutative spacetime. Nucl. Phys. B 2020, 958, 115117. [CrossRef]

16. Zakrzewski, S. A characterization of coboundary poisson lie groups and hopf algebras. Banach Cent. Publ. 1997, 40, 273. [CrossRef]

17. Amelino-Camelia, G.; Freidel, L.; Kowalski-Glikman, J.; Smolin, L. The principle of relative locality, Phys. Rev. D 2011, 84, 084010. [CrossRef]

18. Lizzi, F.; Mercati, F. $\kappa$-Poincaré-comodules, Braided Tensor Products and Noncommutative Quantum Field Theory. Phys. Rev. D 2021, 103, 126009. [CrossRef]

19. Gutt, S. An explicit *-product on the cotangent bundle of a Lie group. Lett. Math. Phys. 1983, 7, 249.

20. Gracia-Bondia, J.M.; Lizzi, F.; Marmo, G.; Vitale, P. Infinitely many star products to play with. J. High Energy Phys. 2002, 4, 26. [CrossRef]

21. Lukierski, J.; Woronowicz, M. New Lie-algebraic and quadratic deformations of Minkowski space from twisted Poincare symmetries. Phys. Lett. B 2006, 633, 116. [CrossRef]

22. Chaichian, M.; Demichev, A.; Presnajder, P. Quantum field theory on noncommutative space-times and the persistence of ultraviolet divergences. Nucl. Phys. B 2000, 567, 360. [CrossRef]

23. Chaichian, M.; Demichev, A.; Presnajder, P.; Tureanu, A. Space-time noncommutativity, discreteness of time and unitarity. Eur. Phys. J. C 2001, 20, 767. [CrossRef]

24. Dolan, B.P.; Gupta, K.S.; Stern, A. Noncommutative BTZ black hole and discrete time. Class. Quant. Grav. 2007, $24,1647$. [CrossRef]

25. Balachandran, A.P.; Martins, A.G.; Teotonio-Sobrinho, P. Discrete time evolution and energy nonconservation in noncommutative physics. J. High Energy Phys. 2007, 5, 66. [CrossRef]

26. Steinacker, H. Split noncommutativity and compactified brane solutions in matrix models. Prog. Theor. Phys. 2011, 126, 613. [CrossRef]

27. Amelino-Camelia, G.; Barcaroli, L.; Bianco, S.; Pensato, L. Planck-scale dual-curvature lensing and spacetime noncommutativity. Adv. High Energy Phys. 2017, 2017, 6075920. [CrossRef]

28. Ćirić, M.D.; Konjik, N.; Samsarov, A. Search for footprints of quantum spacetime in black hole QNM spectrum. arXiv 2019, arXiv:1910.13342

29. Ćirić, M.D.; Konjik, N.; Samsarov, A. Noncommutative scalar field in the nonextremal Reissner-Nordström background: Quasinormal mode spectrum. Phys. Rev. D 2020, 101, 116009. [CrossRef]

30. Ciric, M.D.; Konjik, N.; Kurkov, M.A.; Lizzi, F.; Vitale, P. Noncommutative field theory from angular twist. Phys. Rev. D 2018, 98, 085011. [CrossRef]

31. Lizzi, F.; Vitale, P. Time Discretization From Noncommutativity. Phys. Lett. B 2021, 818, 136372. [CrossRef]

32. Gubitosi, G.; Lizzi, F.; Relancio, J.J.; Vitale, P. Double Quantization. arXiv 2021, arXiv:2112.11401. 